Draft Version SePtember 4, 2018

Preprint typeset using $\mathrm{IAT}_{\mathrm{E}} \mathrm{X}$ style emulateapj v. 5/2/11

\title{
HI-C OBSERVATIONS OF SUNSPOT PENUMBRAL BRIGHT DOTS
}

\author{
Shane E. Alpert \\ Department of Physics and Astronomy, Rice University, Houston, TX, 77005, USA
}

AND

Sanjiv K. Tiwari, Ronald L. Moore, Amy R. Winebarger, and Sabrina L. Savage

NASA Marshall Space Flight Center, ZP 13, Huntsville, AL 35812, USA

Draft version September 4, 2018

\begin{abstract}
We report observations of bright dots (BDs) in a sunspot penumbra using High Resolution Coronal Imager (Hi-C) data in $193 \AA$ and examine their sizes, lifetimes, speeds, and intensities. The sizes of the BDs are on the order of $1^{\prime \prime}$ and are therefore hard to identify in the Atmospheric Imaging Assembly (AIA) $193 \AA$ images, which have $1.2^{\prime \prime}$ spatial resolution, but become readily apparent with Hi-C's five times better spatial resolution. We supplement Hi-C data with data from AIA's $193 \AA$ passband to see the complete lifetime of the BDs that appeared before and/or lasted longer than Hi-C's 3-minute observation period. Most Hi-C BDs show clear lateral movement along penumbral striations, toward or away from the sunspot umbra. Single BDs often interact with other BDs, combining to fade away or brighten. The BDs that do not interact with other BDs tend to have smaller displacements. These BDs are about as numerous but move slower on average than Interface Region Imaging Spectrograph (IRIS) BDs, recently reported by Tian et al. (2014), and the sizes and lifetimes are on the higher end of the distribution of IRIS BDs. Using additional AIA passbands, we compare the lightcurves of the BDs to test whether the Hi-C BDs have transition region (TR) temperature like that of the IRIS BDs. The lightcurves of most Hi-C BDs peak together in different AIA channels indicating that their temperature is likely in the range of the cooler TR $\left(1-4 \times 10^{5} \mathrm{~K}\right)$.
\end{abstract}

Subject headings: Sun: corona — Sun: magnetic fields — Sun: sunspots — Sun: transition region

\section{INTRODUCTION}

In the past few decades, high resolution images from space telescopes have allowed solar astronomers to view and analyze the Sun in unprecedented detail. The data from the High Resolution Coronal Imager (HiC: Kobayashi et al. 2014) yield new information about small-scale structures in the corona and chromospherecorona transition region (TR), including braiding in coronal loops, triggering of subflares in them, and signatures of nanoflares and impulsive heating (e.g., Cirtain et al. 2013, Winebarger et al. 2013, Thalmann et al. 2014; Tiwari et al. 2014). This paper examines a sunspot in $\mathrm{Hi}$ C's field-of-view (FOV) with particular attention to the penumbra. A sunspot consists of the inner, darker umbra, and the outer, lighter (but darker than quiet Sun) penumbra. The umbra has strong nearly vertical magnetic field, with umbral-dots and light-bridges in it as signatures of magnetoconvection (e.g., Sobotka et al. 1993. Weiss 2002, Rimmele 2008, Riethmüller et al. 2013). The magnetic field inclination in the penumbra greatly varies azimuthally at small-scales (Solanki 2003: Mathew et al. 2003; Tiwari et al. 2009; Borrero \& Ichimoto 2011; Scharmer \& Henriques 2012; Tiwari et al. 2013, 2015). The penumbra is known for its filaments, horizontal tendrils that extend outward from the umbra. Penumbral filaments contain a general outflow (the Evershed flow: Evershed 1909); localized up- and downflows at their heads (ends of filaments closer to sunspot center) and tails, respectively. Penumbral filaments have a stretched granule shape and reversed polarity edge field sustained by lateral downflows (Rempel 2012, Tiwari et al. 2013).
In addition to the dynamic features in the photosphere in sunspots e.g., p-modes, inward motion of penumbral grains, moving magnetic features (Harvey \& Harvey 1973), there exist chromospheric and coronal dynamic features in sunspots e.g., umbral flashes and running penumbral waves (Zirin \& Stein 1972), and penumbral jets (Katsukawa et al. 2007; Jurcák \& Katsukawa 2008; Tiwari et al. 2016). There have been only limited investigations of the smaller dynamic events owing to the limited simultaneous temporal and spatial resolution of observations.

Using data from the Interface Region Imaging Spectrograph (IRIS: De Pontieu et al. 2014), Tian et al. (2014) recently discovered bright dots (BDs), another dynamic feature, in the TR of sunspots. The BDs are most numerous in penumbrae but are also present in umbrae and light-bridges. The penumbral BDs observed by IRIS move along the direction of penumbral filaments with speeds of $10-40 \mathrm{~km} \mathrm{~s}^{-1}$, and appear slightly elongated along the filaments, with the two dimensions being 300 $600 \mathrm{~km}$ and $250-450 \mathrm{~km}$, respectively. The lifetimes of the IRIS BDs are mostly less than one minute, some lasting for a few minutes. Many of these BDs were proposed to be signatures of small-scale energy release events at the TR footpoints of coronal magnetic loops.

In this Paper, we report on Hi-C observations of BDs in a sunspot penumbra. We measure and compare the physical characteristics of these Hi-C penumbral BDs to the IRIS penumbral BDs and use different Atmospheric Imaging Assemply (AIA: Lemen et al. 2012) channels to explore the temperature of the Hi-C BDs. 


\section{OBSERVATION AND DATA ANALYSIS}

We use Hi-C $193 \AA$ data taken on July 11, 2012 during 18:52:43-18:55:30 UT. The entire Hi-C FOV is shown in Figure 17. Hi-C data has a cadence of 5.5 seconds and a plate scale of $0.1^{\prime \prime}$ pixel $^{-1}$. The sunspot that is the main focus of this research is centered south-east of disk center, at $\left(-122^{\prime \prime},-325^{\prime \prime}\right)$. A $40^{\prime \prime} \times 40^{\prime \prime} \mathrm{FOV}$ around this point is used for most of the analysis. The blue inset in Figure 1 indicates the Hi-C $40^{\prime \prime} \times 40^{\prime \prime} \mathrm{FOV}$ used in this study, enlargement of which is shown in Figure $1 \mathrm{~b}$. Due to the Hi-C data's short duration and for comparison reasons, supplemental $193 \AA$ data is used from SDO/AIA. AIA has a cadence of 12 seconds ( 24 seconds for UV channels) and a plate scale of $0.6^{\prime \prime}$ pixel $^{-1}$. Figure $1 \mathrm{k}$ is the same FOV and time of observation as Figure $1 \mathrm{~b}$, but shows SDO/AIA $193 \AA$ data. Hi-C BDs are indicated by the smaller contours. Evidently, BDs are present in both umbra and penumbra of the sunspot, most of them being in penumbra. In this Paper, we have focused on the penumbral BDs.

Additional AIA data is used from the 1600, 304, 171, and $211 \AA$ passbands to examine BD visibility at different atmospheric heights/temperatures. To compare the same FOV across instruments, same subregion of the AIA $193 \AA$ image was extracted to match the Hi-C FOV. These AIA $193 \AA$ coordinates were then used for the other AIA channels alignments.

When examining the sunspot, we find small, roundish, BDs. Any compact transient blob that appears brighter than the surrounding area is considered a BD. To identify, track, and measure the BDs easily, we smooth the images $(20 \times 20$ pixels for $\mathrm{Hi}-\mathrm{C}$ and $7 \times 7$ pixels for AIA $)$ and subtract them from the originals. The enhanced images can be seen in comparison to the original images in Movie1. It must be noted that although we used the enhanced images to more easily identify the BDs, all size, intensity, lifetime, speed, and lightcurve measurements were made using the original images.

Figure 2 shows examples of different types of BDs that are seen in the sunspot penumbra. The image sequences display the lateral drift and brightness evolution of the $\mathrm{BDs}$ over the Hi-C observing period. Figure $2 \mathrm{a}$ shows a single stationary BD. Figure $2 \mathrm{~b}$ shows a single BD that moves away from the umbra. Figure 25 shows two separate BDs moving towards each other before combining to form one larger, brighter BD. Figure $2 \mathrm{~d}$ shows a triplesystem of BDs; a larger BD splits to become two separate, smaller BDs and one of these then combines with a third BD. Unlike the combined, large BDs in Figure 2 , the BDs in Figure $2 \mathrm{~d}$ fade as they merge. It must be noted that all BD motion, if motion is seen at all, is along the direction of the penumbral filamentary structures. During its lifetime, a BD may move inward and outward, i.e., oscillatory motion is commonly seen. To look at all of the BDs in the sunspot, see Movie1.

We tracked $30 \mathrm{BDs}$ by eye and characterized them. To get the best sense of the movement and overall changes of the BDs, we picked one at a time and tracked throughout the length of the movie, combining $\mathrm{Hi}-\mathrm{C}$ and AIA data. We repeated this for a few movie cycles, and for each of 30 BDs. Note that for the BDs that separate into smaller BDs, a variety of motion is seen after the split; the individual BDs may move apart, remain stationary, one moves while the other remains stationary, or they may recombine to either form a larger, brighter BD (Figure 2k) or fade away (Figure 2d). The longestlived BDs tend to be the ones that are large, bright, and isolated (no interaction with other BDs).

\section{RESULTS AND DISCUSSION}

Figure 3 shows histograms of the size, intensity, lifetime, and speed measurements of the 30 BDs that we selected in this sunspot penumbra. This figure is similar to Figure 3 of Tian et al. (2014) and we replicate their measurement methods closely in order to make the best comparisons between the properties of IRIS BDs and our BDs. Figures 3 a,b,c depict how the size was determined. Noticing the slightly non-circular shape of the BDs and in being consistent with the techniques used by Tian et al. (2014), we measured length and width for each BD. The measurement was taken when the BD was the brightest. Figure 3 a shows one example $\mathrm{BD}$ in the normal intensity image. The length, measured along the black line, is in the local direction of the penumbral striation. The width is measured along the red line, perpendicular to the length cut. Figure $3 \mathrm{~b}$ shows the same dot as in 3 a, but from the enhanced image, which is used to determine the direction and placement of the dots.

In Figure 3r, we show plots of intensity along each cut fitted with a Gaussian. Please note that a non-constant background is used when fitting the Gaussian. The size of the dot (length and width) is the full-width at halfmaximum of the Gaussian. Figure $3 \mathrm{~d}$ displays the distribution of $30 \mathrm{BD}$ lengths and widths; the length has a mean of $557 \mathrm{~km}$ and standard deviation of $252 \mathrm{~km}$ while the width has a mean of $472 \mathrm{~km}$ and a standard deviation of $185 \mathrm{~km}$. Figure $3 \mathrm{e}$ is the length-to-width ratio, showing that about half of the BDs are longer than they are wide. On average, BDs are not significantly larger in their direction of motion than in the orthogonal direction. Figure 3 f shows the intensity enhancement of each BD, measured as the brightness of the brightest part of the dot divided by the background brightness, which is estimated by averaging intensities of many pixels surrounding the BD. Average BDs are just under twice brighter than their surroundings.

The histogram in Figure $3 \mathrm{~s}$ represents the lifetime of the BDs, tracked manually and supplemented with AIA $193 \AA$ data for the longer-lasting BDs. Most of them last 1-5 minutes, but several survive for 12-14 minutes. The longer-lasting BDs are normally the bright, large, stationary ones and are closer to the sunspot umbra.

Figure 3 h displays a histogram of the speed of the BDs. To calculate the speed of the BDs, we track the center of the BD as it moves. The tracking is done as follows. The initial location of the center of the $\mathrm{BD}$ is recorded and then the image is advanced in time. The location is then recorded again. The velocity is calculated by dividing the distance the center of the $\mathrm{BD}$ moved by the time between recordings. The image was then advanced again and this process is repeated until the BD disappears or merges with another BD. Because the lifetime of the BDs is variable, the time between recordings varies, but as a general rule, the number of recordings is maximized (minimizing the time between each recording) but there are at least 11 seconds between each recording. The 

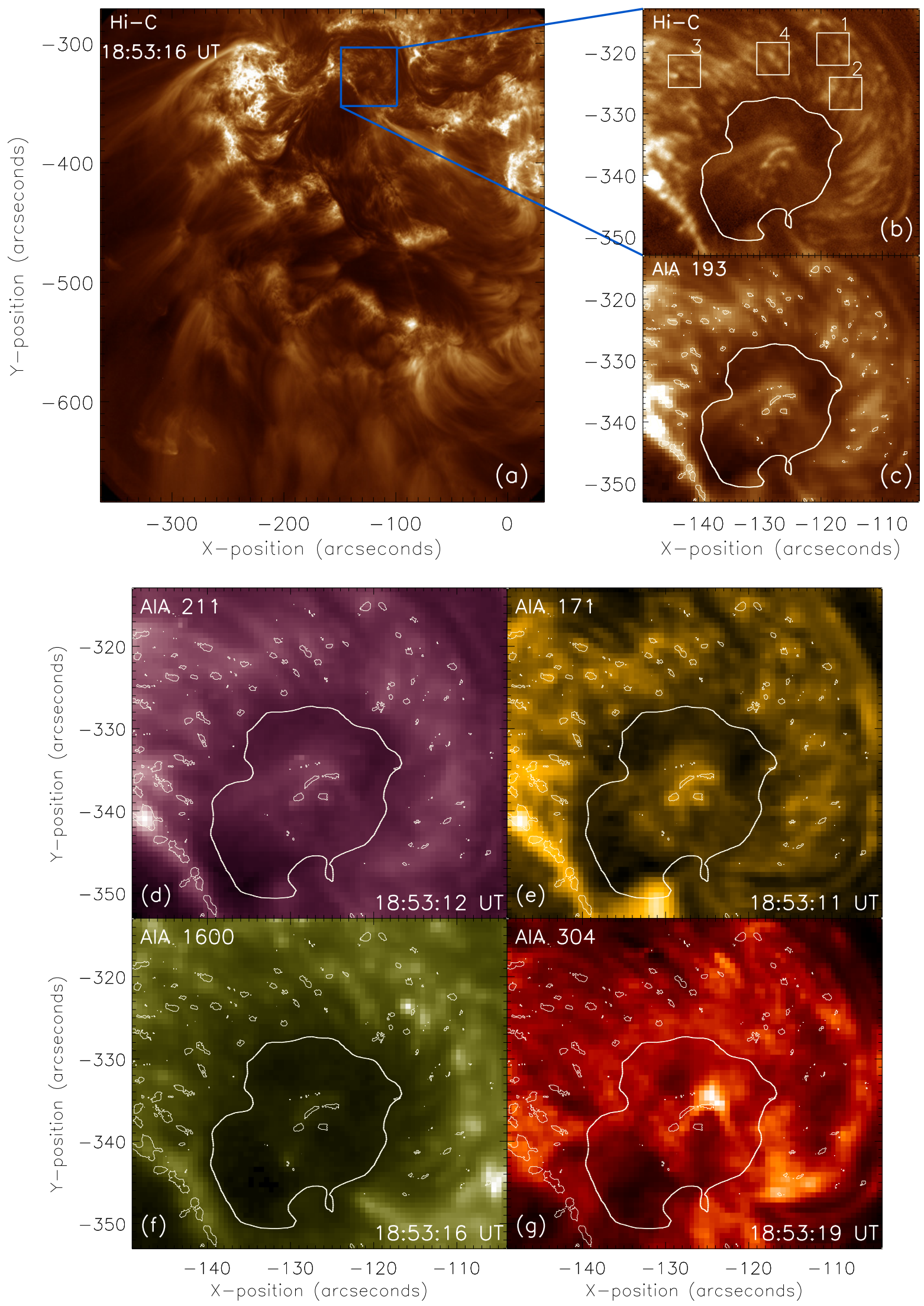

FIG. 1.- (a) Full FOV of the Hi-C, $\sim 400^{\prime \prime} \times 400^{\prime \prime}$. The blue box covers the sunspot studied here. (b) depicts a close-up view $\left(\sim 40^{\prime \prime} \times\right.$ $\left.40^{\prime \prime}\right)$ of the sunspot as seen by Hi-C. The white boxes 1, 2, 3, and 4 outline the locations of example BDs shown in Figure 2 a, b, c, and d, respectively. (c) is the same FOV and time as (b), but from the AIA $193 \AA$ movie. (d-g) The same $40^{\prime \prime} \times 40^{\prime \prime}$ FOV as in (b) and (c), but in other AIA bands. (d), (e), (f) and (g) are 211, 171, 1600 and $304 \AA$, respectively. Smaller contours outline Hi-C BDs and are from an enhanced Hi-C image. The large contour roughly outlines the sunspot umbra; most of the outside displayed area being penumbra. 


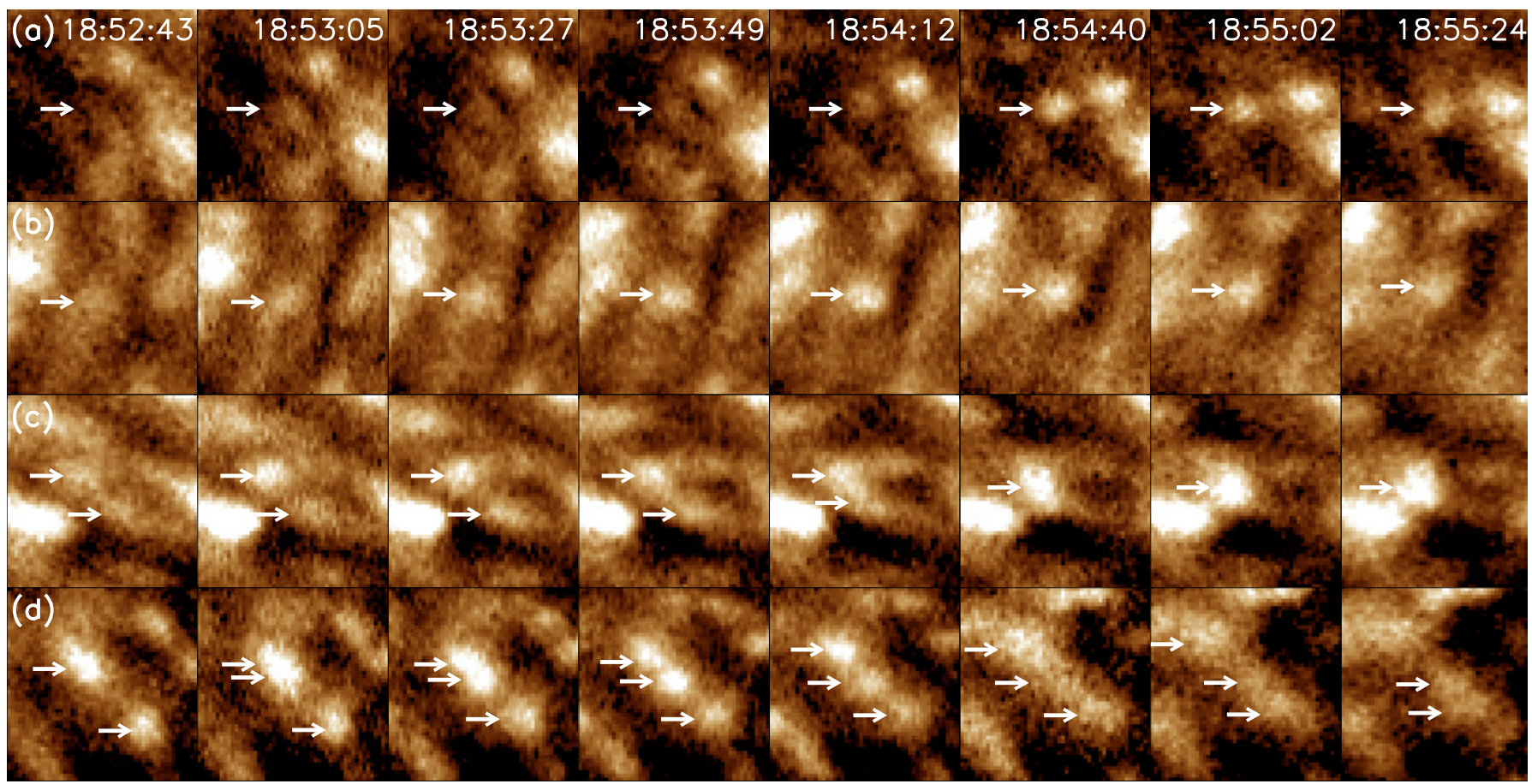

FIG. 2.- Examples of four different types of Hi-C penumbral BDs; how they evolve over a three minute period, with arrows pointing to the centers of the BDs. (a) A single BD is stationary while brightening and then fading. (b) A single BD moves laterally outward from sunspot center while brightening and then fading. (c) Two BDs move toward each other and combine into a larger, brighter BD. (d) A system of three BDs. A large BD on the upper left separates into two BDs, with the lower one moving laterally towards sunspot center. A single $\mathrm{BD}$ on the lower right moves laterally away from sunspot center. The inward moving BD and the outward moving BD merge as they fade. The size of each panel is $5^{\prime \prime} \times 5^{\prime \prime}$, and their locations are outlined in Figure 1 (b) by four white boxes numbered $1,2,3$, and 4 . 

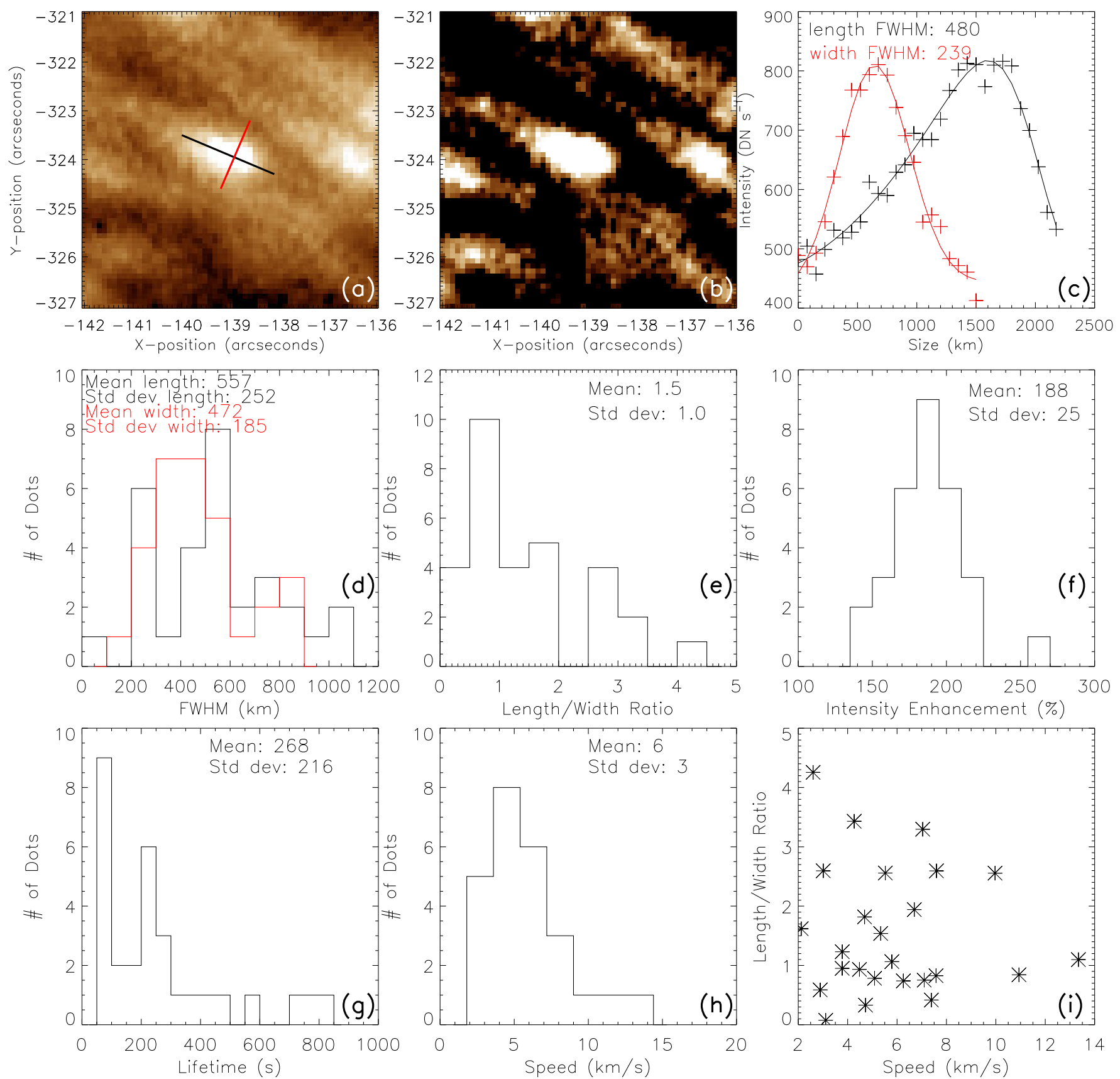

FIG. 3.- Characteristics of Hi-C penumbral BDs. (a) Two cuts are used to measure the size of each BD. Black is for measurement of length (in the direction along the penumbral striations) and red is for measurement of width (perpendicular to length). (b) Same BD as in a), but enhanced image shown. (c) Example of BD size measurement; intensity is shown as a function of both length and width. A Gaussian fit is made to each cut and the FWHM is taken as the length/width. (d) Histogram of all length and width measurements. (e) Histogram of the ratio of length to width. (f) Histogram of how much brighter a BD is than its surrounding background. (g) Histogram of lifetimes of the BDs. (h) Histogram of the speed of the BDs. (i) Scatter plot of length/width ratio as a function of BD speed.

average velocity of a $\mathrm{BD}$ is the average of these multiple velocity measurements. The speed was measured for only $25 \mathrm{BDs}$ because there were not enough data points for five of the BDs. Figure 3 is a scatterplot between the speed and the ratio of length-to-width of the BDs. We plot this figure in an attempt to find a correlation between how long a BD is relative to how fast it is moving (the thought being that faster BDs might be elongated in the radial direction), but evidently there is no clear relationship; there is perhaps a slight tendency for faster BDs to have less elongation.
To investigate the formation mechanism and temperature of these BDs, we obtained their lightcurves from five AIA channels. Figure 4 shows the lightcurves of four of our BDs. These lightcurves are made by tracking the BD from when it first begins to brighten, through peak intensity, and ending when it fades from view or gets dim enough. To measure the intensity, we first find the target $\mathrm{BD}$ using the Hi-C $193 \AA$ images and then match the location to the AIA $193 \AA$ images. We center a circle on the BD's brightest part and measure the intensity inside. The frame is advanced and the intensity 
TABLE 1

COMPARISON OF PENUMBRAL BD AVERAGE QUANTITIES MEASURED IN TIAN ET AL. (2014) AND IN THIS WORK.

\begin{tabular}{|l|c|r|}
\hline & Tian et al. & This research \\
\hline Length $(\mathrm{km})$ & 439 & 558 \\
\hline Width $(\mathrm{km})$ & 352 & 472 \\
\hline Intensity Enhancement $(\%)$ & 319 & 188 \\
\hline Lifetime $(\mathrm{s})$ & 41 & 268 \\
\hline Speed $(\mathrm{km} / \mathrm{s})$ & 22 & 6 \\
\hline
\end{tabular}

is measured for the new time, again with the circle centered on the brightest part of the $\mathrm{BD}$. In this way, we track the BD's peak brightness over its lifetime or over its brightest peak during its lifetime. This intensity is then normalized to the highest intensity over the period.

We use four AIA wavelengths in addition to the $193 \AA$ : 211, 171, 1600, and $304 \AA$. The AIA 171, 193, and 211 channels are typically coronal, as their response functions peak at temperatures of roughly $1,1.5$, and $2.5 \mathrm{MK}$, respectively (Lemen et al. 2012). However, all of these channels have cooler-TR lines in the passband and hence have some response in the cooler-TR temperature range (e.g., O'Dwyer et al. 2010). The 304 and 1600 channels both respond to cooler-TR emission. If the BDs have cooler-TR temperature then these different wavelength channels, which all have some sensitivity to emission from plasma at TR temperature, will peak at the same time (Winebarger et al. 2013). If the BDs are heated to (and cooling from) coronal temperatures, then the different channels will not peak at the same time. Figure 4 shows the lightcurves of a few representative BDs, showing that the different wavelengths do peak at the same time.

These lightcurves indicate that the BD plasma is at cooler-TR temperatures $\left(1-4 \times 10^{5} \mathrm{~K}\right)$; its appearance in AIA coronal channels is due to leakage of low temperature emission into the predominantly coronal channels. Note that the lightcurves before and/or after the peak brightness need not necessarily be in phase.

After determining the characteristics of the BDs that we see with $\mathrm{Hi}-\mathrm{C}$, we compare them to the BDs seen by Tian et al. (2014) using the IRIS telescope. Table 1 compares our measured $\mathrm{BD}$ values with those found in Tian et al. (2014). All listed values are averages.

Tian et al. (2014) did not calculate average speed because they measured dot velocities, i.e., they included the direction the dot moves in their data. In this work, we measure the speed of the $\mathrm{BD}$ as the distance traveled by the center of the $\mathrm{BD}$ over time, without reference to the direction of travel. We deduce from Table 1 that our BDs are, on average, larger, longer-lived, and slower than the dots seen in the TR with IRIS. These results and our finding from the lightcurves that the Hi-C BDs have cooler-TR temperature implies that Hi-C detects only the brighter BDs seen by IRIS. The smaller and dimmer BDs are probably masked by the Fe XII emission of the overlying corona.

Based on our analysis, there are several different mechanisms that could be the cause of these BDs. Because the BDs are at the feet of coronal loops, cooled plasma that flows down along the loops could hit the lower, denser atmosphere and create shocks that locally heat the plasma and cause an increase in intensity: this requires supersonic downflow $\left(\sim 200 \mathrm{~km} \mathrm{~s}^{-1}\right)$ (Kleint et al. 2014). The heating could also be due to the intricate, interlaced magnetic fields of the penumbra repeatedly reconnecting; the radially inward/outward motion of BDs might result from sliding reconnection between the two inclined penumbral field components (spines and bulk of penumbral filaments). Also using Hi-C data, Régnier et al. (2014) found EUV bright dots at the edges of active regions which have a characteristic diameter of $680 \mathrm{~km}$ and a duration of $25 \mathrm{~s}$; they proposed impulsive energy release in the coronal loops (Parker 1988) rooted in the dots being responsible for those dots. Ubiquitous chromospheric bright grains have also been observed in coronal holes and the quiet Sun, also on the order of $1^{\prime \prime}$ in diameter and lasting 1.5-2.5 minutes (e.g., Martínez-Sykora et al. 2015).

Running penumbral waves (Zirin \& Stein 1972, Moore 1973: Bloomfield et al. 2007), which are driven by photospheric p-mode oscillations, could perhaps be responsible for the oscillatory motion of some of the BDs. These waves could cause the BDs to move along field lines rooted in the penumbra. However, there are very slow moving and stationary BDs present near the BDs that oscillate; this casts doubt on running penumbral waves as the cause of all of our BDs.

The slowest moving or stationary BDs might be at the heads of penumbral filaments (Tiwari et al. 2013), also known as penumbral grains (Muller 1973; Rimmele \& Marino 2006). Those BDs might then be the result of opposite polarity fields at the sides of filaments reconnecting with the surrounding spines. This mechanism would also create penumbral jets (Katsukawa et al.|2007), as proposed by Tiwari et al. (2016). Thus, some penumbral jets and BDs perhaps have the same location and same cause. This proposal is similar to the one given by Vissers et al. (2015) that some penumbral BDs are the heating signatures of penumbral jets. However, from Figure 2 of Tiwari et al. (2016) we find evidence that most of the Hi-C penumbral BDs do not coincide with penumbral jets. As pointed out by Tian et al. (2014), some of the BDs concievably could be produced by moving magnetic features (Zhang et al. 2003 Ravindra 2006 Sainz Dalda \& Bellot Rubio 2008).

\section{CONCLUSIONS}

We observe BDs in the penumbra of a sunspot using $193 \AA$ data from the Hi-C. Their lateral motion is inward or outward from the sunspot center, following the direction of penumbral filaments. We compare our BDs to those found by Tian et al. (2014) in the TR using the IRIS telescope. Our BDs are 300-800 km long and $300-600 \mathrm{~km}$ wide, last 1-5 minutes or longer, are double the intensity of the surrounding regions, and move 3-15 $\mathrm{km} \mathrm{s}^{-1}$. In general, they are larger, longer-lived, and slower than the IRIS BDs. Based on the lightcurves obtained using supplemental AIA data, most of the plasma in our BDs is probably at cooler-TR temperature, but the data is widely varied and subject to resolution and temporal constraints. The cause of these BDs is an open question. They are conceivably a consequence of plasma downflow impacting the TR or upper-chromosphere density, or of reconnection between two inclined components of penumbral magnetic field (more vertical spine field 
Dot 2

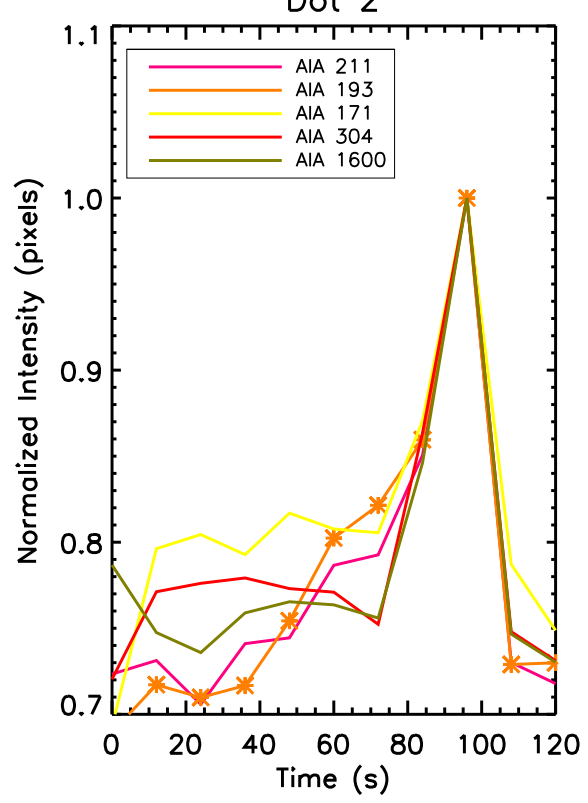

Dot 7

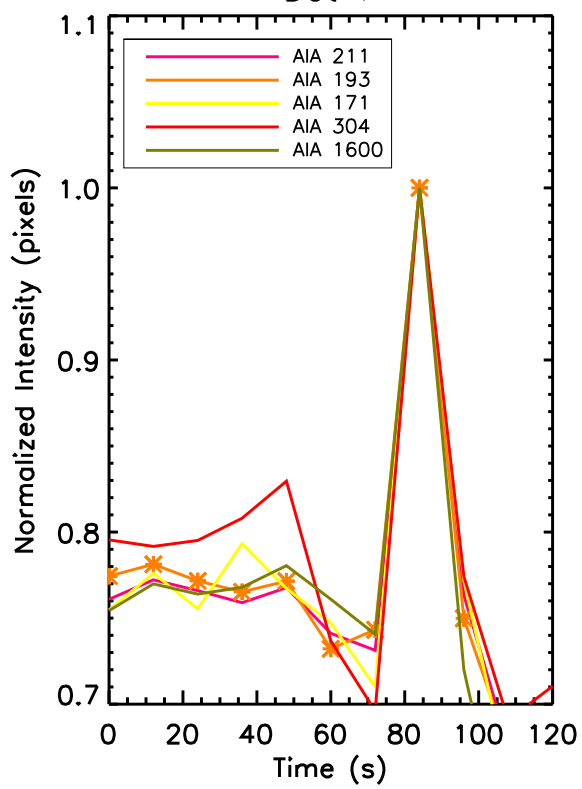

Dot 5

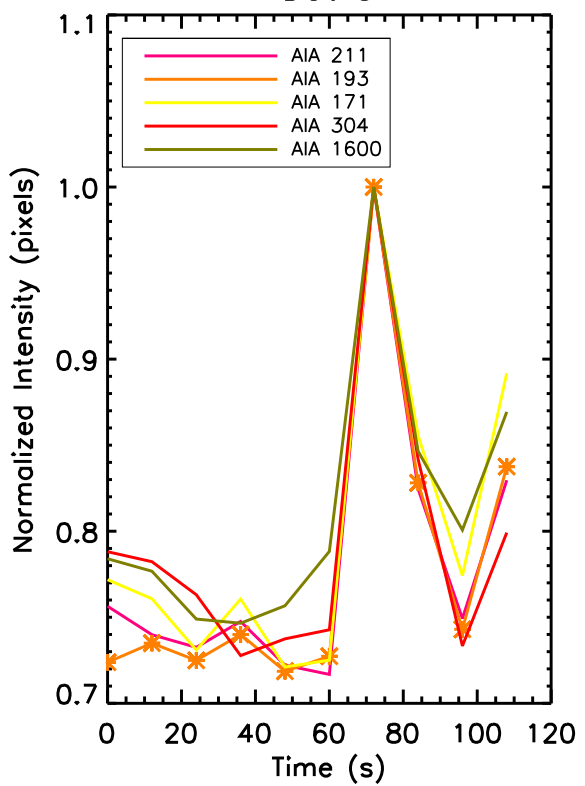

Dot 26

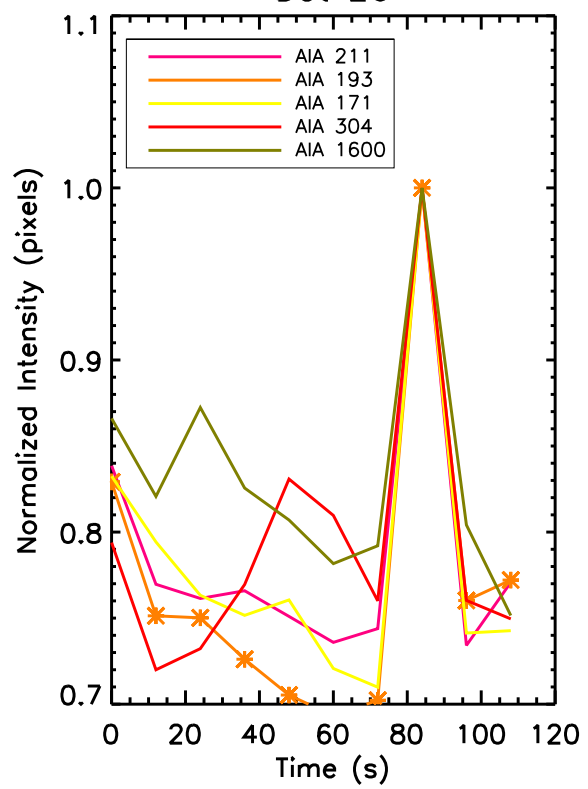

FIG. 4.- Normalized lightcurves for four example BDs; from a set of five AIA bands. Each set of lightcurves is from tracking the brightest part of the BD as it drifts laterally during its peak brightening. As denoted in each panel by different colored lines, orange is for 193, pink is for 211, yellow is for 171, green is for 1600 , and red is for $304 \AA$. Please note that the time on $\mathrm{x}$-axis is based on the AIA 193 channel, the closest available data in time for other channels is taken at each time step. Therefore a few seconds difference in the observation of different channels is not seen. Asterisks on 193 lightcurves mark data points. 
and more horizontal field in the bulk of penumbral filaments), perhaps sometimes triggered by running penumbral waves. The Hi-C BDs belong to the class of TR penumbral BDs found in IRIS data by Tian et al. (2014), and have possible counterparts at the edges of active regions in the corona and/or TR (Régnier et al. 2014).

This work is supported by NSF Grant No. AGS1157027, a Research Experience for Undergraduates grant. These BDs were reported in 2014 at the LWS Science Meeting and at the AGU meeting on November
2-6 and December 15-19, respectively. Thanks goes to Hui Tian for helpful discussion on BDs and the methods of measurement. SKT is supported by an appointment to the NASA Postdoctoral Program at the NASA MSFC, administered by USRA through a contract with NASA. RLM and AW were supported by funding from the LWS TRT Program of the Heliophysics Division of NASA's SMD. SLS is supported through the Hinode project office as part of NASA SMD's Solar Terrestrial Probes Program.

\section{REFERENCES}

Bloomfield, D. S., Lagg, A., \& Solanki, S. K. 2007, ApJ, 671, 1005

Borrero, J. M., \& Ichimoto, K. 2011, Living Reviews In Solar Physics, 8, 4

Cirtain, J. W., Golub, L., Winebarger, A. R., et al. 2013, Nature, 493, 501

De Pontieu, B., Title, A. M., Lemen, J. R., et al. 2014, Sol. Phys., 289, 2733

Evershed, J. 1909, MNRAS, 69, 454

Harvey, K., \& Harvey, J. 1973, Sol. Phys., 28, 61

Jurčák, J., \& Katsukawa, Y. 2008, A\&A, 488, L33

Katsukawa, Y., Berger, T. E., Ichimoto, K., et al. 2007, Science, 318, 1594

Kleint, L., Antolin, P., Tian, H., et al. 2014, ApJ, 789, L42

Kobayashi, K., Cirtain, J., Winebarger, A. R., et al. 2014, Sol. Phys., 289, 4393

Lemen, J. R., Title, A. M., Akin, D. J., et al. 2012, Sol. Phys. 275,17

Martínez-Sykora, J., Rouppe van der Voort, L., Carlsson, M., et al. 2015, ApJ, 803, 44

Mathew, S. K., Lagg, A., Solanki, S. K., et al. 2003, A\&A, 410, 695

Moore, R. L. 1973, Sol. Phys., 30, 403

Muller, R. 1973, Sol. Phys., 29, 55

O’Dwyer, B., Del Zanna, G., Mason, H. E., Weber, M. A., \& Tripathi, D. 2010, A\&A, 521, A21

Parker, E. N. 1988, ApJ, 330, 474

Ravindra, B. 2006, Sol. Phys., 237, 297

Régnier, S., Alexander, C. E., Walsh, R. W., et al. 2014, ApJ, 784,134
Rempel, M. 2012, ApJ, 750, 62

Riethmüller, T. L., Solanki, S. K., van Noort, M., \& Tiwari, S. K. 2013, A\&A, 554, A53

Rimmele, T. 2008, ApJ, 672, 684

Rimmele, T., \& Marino, J. 2006, ApJ, 646, 593

Sainz Dalda, A., \& Bellot Rubio, L. R. 2008, A\&A, 481, L21

Scharmer, G. B., \& Henriques, V. M. J. 2012, A\&A, 540, A19

Sobotka, M., Bonet, J. A., \& Vazquez, M. 1993, ApJ, 415, 832

Solanki, S. K. 2003, A\&A Rev., 11, 153

Thalmann, J. K., Tiwari, S. K., \& Wiegelmann, T. 2014, ApJ, 780,102

Tian, H., Kleint, L., Peter, H., et al. 2014, ApJ, 790, L29

Tiwari, S. K., Alexander, C. E., Winebarger, A. R., \& Moore, R. L. 2014, ApJ, 795, L24

Tiwari, S. K., Moore, R. L., Winebarger, A. R., \& Alpert, S. E. 2016, ApJ, 816, 92

Tiwari, S. K., van Noort, M., Lagg, A., \& Solanki, S. K. 2013, A\&A, 557, A25

Tiwari, S. K., van Noort, M., Solanki, S. K., \& Lagg, A. 2015, A\&A, 583, A119

Tiwari, S. K., Venkatakrishnan, P., \& Sankarasubramanian, K. 2009, ApJ, 702, L133

Vissers, G. J. M., Rouppe van der Voort, L. H. M., \& Carlsson, M. 2015, ApJ, 811, L33

Weiss, N. O. 2002, Astronomische Nachrichten, 323, 371

Winebarger, A. R., Walsh, R. W., Moore, R., et al. 2013, ApJ, 771,21

Zhang, J., Solanki, S. K., \& Wang, J. 2003, A\&A, 399, 755

Zirin, H., \& Stein, A. 1972, ApJ, 178, L85 